\title{
Emulating the Perceptual System of the Brain for the Purpose of Sensor Fusion
}

\author{
Rosemarie Velik, Member, IEEE, Roland Lang, Member, IEEE, Dietmar Bruckner, Member, IEEE, \\ and Tobias Deutsch, Member, IEEE
}

\begin{abstract}
This work presents a bionic model derived from research findings about the perceptual system of the human brain to build next generation intelligent sensor fusion systems. Therefore, a new information processing principle called neuro-symbolic information processing is introduced. According to this method, sensory data are processed by so-called neuro-symbolic networks. The basic processing units of neuro-symbolic networks are neurosymbols. Correlations between neuro-symbols of a neurosymbolic network can be learned from examples. Perception is based on sensor data as well as on interaction with cognitive processes like focus of attention, memory, and knowledge. Additionally, a mechanism for evaluating perception by emotions is suggested.
\end{abstract}

Keywords - Humanlike Perception, Sensor Fusion, Neuro-symbolic Networks, Learning, Knowledge-based Systems, Focus of Attention.

\section{INTRODUCTION}

$\mathrm{T}$ HE human brain is a highly complex system, which is capable of performing a huge range of diverse tasks. One capability of the brain is to process information coming from thousands and thousands of sensory receptors and integrating this information into a unified perception of the environment. Up to now, technical systems used for machine perception can by far not compete with their biological archetype. Having available a technical system, which is capable of perceiving objects, events, and situations in a similar efficient manner as the brain does, would be very valuable for a wide range of applications. Examples for applications are automatic surveillance systems in buildings and autonomous robots. To perceive objects, events, and situations in an environment, sensors of various types are necessary. The challenge that has to be fenced for perceptive tasks is the merging and the interpretation of sensory data from various sources. The aim of this paper is to introduce a model for integrating and interpreting such data. As humans can perceive their environment very effectively, the perceptual system of the brain serves as archetype for model development. Particularly, research findings from neuroscience and neuro-psychology are the guides in the development process.

Rosemarie Velik, Roland Lang, Dietmar Bruckner, and Tobias Deutsch are with the Vienna University of Technology, Institute of Computer Technology, 1040 Vienna, Austria. \{velik, langr, bruckner, deutsch\}@ict.tuwien.ac.at

\section{StATE OF THE ART}

There have already been made various attempts to merge information coming from various sensory sources. The research area generally first mentioned when discussing such tasks is the area of sensor fusion. In literature, there cannot be found only one but various definitions of the term sensor fusion. Principally, sensor fusion is concerned with the combination of sensor data or data derived from sensory data in order to produce enhanced data in form of an internal representation of the process environment. The achievements of sensor fusion are robustness, extended spatial and temporal coverage, increased confidence, reduced ambiguity and uncertainty, and improved resolution [8]. The research field of sensor data fusion is relatively recent and dynamic. Therefore, a standard terminology has not yet evolved. There are widely used the terms "sensor fusion", "sensor integration", "data fusion", "information fusion", "multi-sensor data fusion", and "multi-sensor integration" [2], [28]. Data for sensor fusion can come from one single sensor taken from multiple measurements subsequently at different instants of time, from multiple sensors of identical types, or from sensors of different types. Applications for fusion are various and range from measurement engineering and production engineering over robotics and navigation to medicine technology and military applications [19], [25]. There have been proposed various models for sensor fusion. However, up to now, the selection of a model strongly depends on the special application. There does not yet exist a model for sensor fusion that is generally accepted. Many researchers even point out that it is very unlikely that one technique or architecture will provide a uniformly, superior solution [9].

In [21], it is pointed out that it generally accepted that sensor fusion in the perceptual system of the human brain is of far superior quality than sensor fusion achieved with existing mathematical methods. Therefore, it seems to be particularly useful to study biological principles of sensor fusion. Such studies can on the one hand lead to better technical models for sensor fusion and on the other hand to a better understanding of how perception is performed in the brain. Sensor fusion based on models derived from biology is called biological sensor fusion. Models for biological sensor fusion based on neural networks have been proposed in [5] and [15]. There have also been made attempts to merge sensory data by transforming sensor data into symbols.

Approaches to process sensor information symbolically have been described by [3], [13], [18], [22], and [23] 
There are often suggested layered architectures for this purpose. Fusion of sensor data from a set of heterogeneous or homogeneous sensors, soft sensors, and history values of sensor data is generally called direct fusion. However, there does also exist indirect fusion, which uses information sources like prior knowledge about the environment and human input. Furthermore, it is possible to fuse the outputs of direct and indirect fusion. In literature, different models for such hybrid systems have been described: [2], [4], [7], [10], [26].

\section{CHARACTERISTICS OF HUMAN PERCEPTION}

The aim of this paper is to introduce a new model for sensor fusion for the purpose of machine perception, which is based on research findings about the perceptual system of the human brain. To develop such a model, in a first step, characteristics of human perception have to be identified. Figure 1 gives an overview about important mechanisms and factors that form and influence human perception. The mentioned characteristics are derived from research results of neuroscience and neuropsychology about the perceptual system of the human brain.

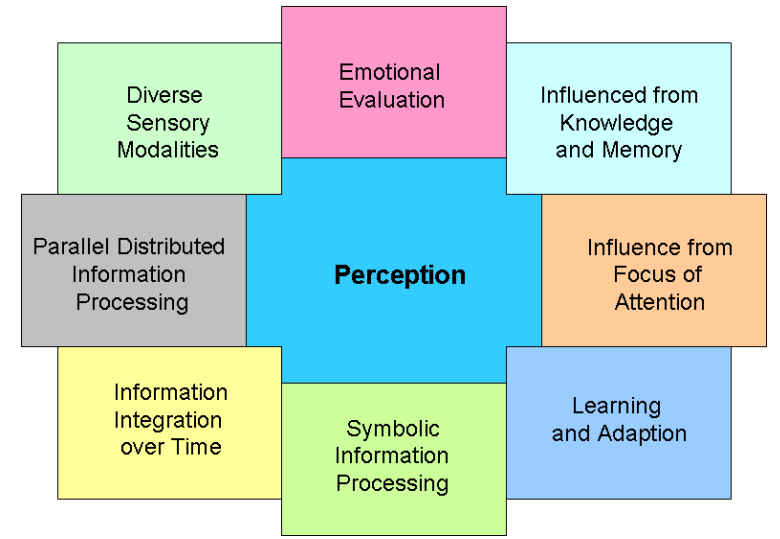

Fig. 1. Characteristics of Human Perception

\section{Diverse Sensory Modalities}

To perceive the external environment, our brain uses multiple sources of sensory information derived from several different modalities including vision, touch, and audition. The combination and integration of multiple sources of sensory information is the key to robust perception [10].

\section{Parallel Distributed Information Processing}

As just outlined, for perception, information from various sources is processed. However, the perceptual system is no unitary central unit that processes all information in one step. Instead, sensory information is processed in parallel [20].

\section{Information Integration over Time}

To perceive objects, events, and situations in an environment, single-moment snapshots of sensory information provided by different modalities is not always sufficient for unambiguous perception. The course and the succession of sensory signals over time is of importance [24].

\section{Symbolic Information Processing}

In the human brain, perceptual information from different modalities is processed by interacting neurons. However, humans do not think in terms of action potential and firing nerve cells but in terms of symbols. Mental processes are often considered as a process of symbol manipulation [11].

\section{Learning and Adaptation}

The perceptual system of the human brain is not fully developed at birth. Although certain patterns need to be predefined by the genetic code, lots of concepts and correlations concerning perception are learned during lifetime [20].

\section{Influence from Focus of Attention}

According to the hypothesis of focused attention, what we see is determined by what we attend to. At every moment, the environment presents far more perceptual information than can be effectively processed. Attention can be used to select relevant information and to ignore irrelevant or interfering information. Instead of trying to process all objects simultaneously, processing is limited to one object in a certain area of space at a time [17].

\section{Influence from Knowledge and Memory}

Perception is facilitated by knowledge. Prior knowledge is often required for interpreting ambiguous sensory signals. Much of what we take for granted as the way the world is - as we perceive it - is in fact what we have learned about the world - as we remember it. Much of what we take for perception is in fact memory. We frequently see things that are not there, simply because we expect them to be there [12].

\section{Emotional Evaluation}

For perception, there are most often only considered the detection and the processing of stimuli from the external environment. However, the perception of objects, events, and situations makes little sense if we do not know what influence they have on our body. In the human brain, an evaluation of perceptual images is performed by emotions. The basic function of emotions in perception is to classify objects, events, and scenarios as good or bad. Emotions are necessary, to react adequately on perceived objects, events, and situations [27].

\section{BIONIC MODEL FOR PERCEPTION}

\section{A. Neuro-symbolic Information Processing}

To fulfill the first five characteristics mentioned in the last section, a new concept of information processing is introduced, which is called neuro-symbolic information processing. This concept is outlined in the following.

\section{Neuro-symbols as Basic Processing Units}

The basic information processing units for neuro-symbolic information processing are so-called neuro-symbols. The inspiration for the usage of such neuro-symbols came from the following observations made from neuroscience and neuro-psychology: It is generally accepted that information in the human brain is processed by neurons. These neurons are interconnected and exchange information with each other via a special neural code. However, humans do not think in terms of action potentials and firing nerve cells, but they think in terms of symbols. According to the theory of symbolic systems, the mind is a symbol system and cognition is symbol manipulation. Examples for symbols are objects, characters, figures, sounds, or colors used to represent 
abstract ideas and concepts. Each symbol is associated with other symbols. Symbol manipulation offers the possibility to generate complex behavior [11].

To sum up, neurons could be regarded as basic information processing on a physiological basis and symbols as information processing units on a more abstract level. An interesting question is if there exists an interface between these two levels of abstraction. Actually, there have been found neurons in the brain, which respond exclusively to certain perceptual images. For example, there have been found neurons in the secondary visual cortex that respond exclusively to the perception of faces. This fact inspired the usage of neurosymbols. Neuro-symbols stand for perceptual images. Perceptual images are for example a face, a person, or a voice. A neuro-symbol has an activation state and is activated if the perceptual image that it represents is perceived in the environment. Neuro-symbols have a certain number of inputs and one output (see figure 2). Via the input, information about the activation state of other neuro-symbols or triggered sensory receptors is received. All incoming activation states are summed up. If this sum exceeds a certain threshold, the neuro-symbol is activated. The information about its activation state is transmitted via the output to other neuro-symbols it is connected to. To be able to consider also input data arriving asynchronously and the succession of sensor signals over time, there exist mechanisms in neuro-symbols that allow the processing of input data arriving within a certain time window or to consider certain successions of incoming data.

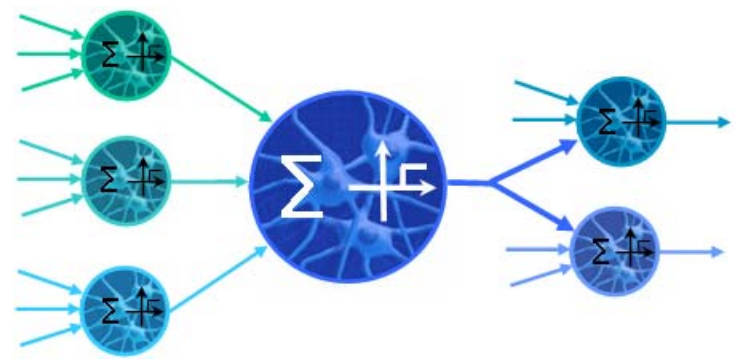

Fig. 2. Function Principle of a Neuro-symbol

\section{Neuro-symbolic Networks}

To perform complex perceptive tasks, a certain number of neuro-symbols have to be interconnected. An important question is how these neuro-symbols should best be structured. As mentioned above, the structural organization of the perceptual system of the brain is taken as archetype. According to [20], the perceptual system of the brain has a cerebral organization as depicted in figure 3. Perception starts with information coming from sensory receptors. Afterwards, this information is processed in three levels, which are referred to as primary cortex, secondary cortex, and tertiary cortex. Each sensory modality of human perception has its own primary and sencondary cortex. This means that in the first two levels, information of different sensory modalities is processed separately and in parallel. In the tertiary cortex, information coming from all sensory modalities is merged. This results in a unified multimodal perception. Examples for perceptual images in the primary cortex of the visual system would be simple features like edges, lines, or movement. Examples for the result of information processing in the primary cortex of the auditory system are sounds of a certain frequency. A perceptual image of the secondary cortex of the visual system could be a face, a person, or an object. Examples for perceptual images in the acoustic system on this level would be a melody or a voice. An example for a task performed in the tertiary cortex would be to merge the perceptual visual image of a face and the perceptual auditory image of a voice to the perception that a person is currently talking. The somatosensory system of the brain (commonly known as tactile system) comprises in fact a whole group of sensory systems, including the cutaneous sensations, proprioception, and kinesthesis.

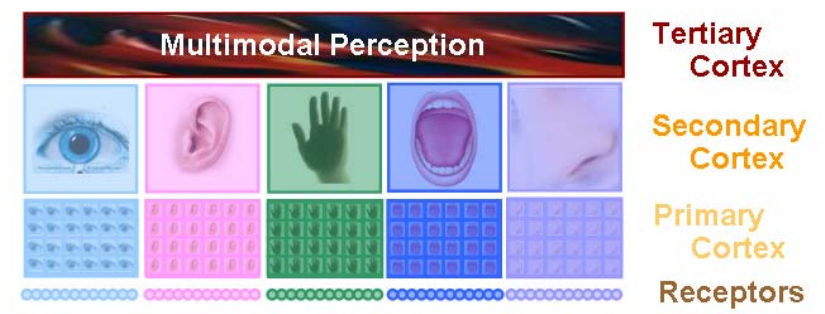

Fig. 3. Cerebral Organization of the Perceptual System of the Brain

In accordance to this organization of the perceptual system of the brain, in the proposed model, neuro-symbols are structured to so-called neuro-symbolic networks (see figure 4). Due to ease of writing, in the following, neurosymbols are also simply referred to as symbols. In a first processing step, so-called feature symbols are extracted out of sensory raw data. Information processing in this level correlates with information processing performed in the primary cortex of the brain. In the next two steps, feature symbols are combined to sub-unimodal and unimodal symbols. These two levels correspond to the function of the secondary cortex of the brain. In connection with the somatosensory system of the brain it was mentioned that a sensory modality can consist of further sub-modalities. Therefore, there can exist a subunimodal level between the feature level and the unimodal level. By processing information in these two steps, different sensor types, for example different video cameras, cameras mounted at different positions, or different tactile sensors like floor sensors, motion detectors, and light barriers, can be merged to one unified modality. On the multimodal level, information from all unimodal symbols is merged to multimodal symbols. Examples and concept clarifications concerning the usage of neuro-symbols for concrete perceptive tasks can be found in [29] and [30].

\section{Learning in Neuro-symbolic Networks}

In neuro-symbolic networks, a great part of the information does not only lie in the perceptual neurosymbols themselves but in the connections between neurosymbols. Therefore, the question how to determine what neuro-symbols shall be connected and exchange information is very important. To answer this question, again, research findings from neuroscience are exploited. As outlined in [20], higher cortical layers can only evolve if lower levels have already developed. Besides this, certain correlations have to be already innate and therefore be predefined by genes. Guided by this description, in the 


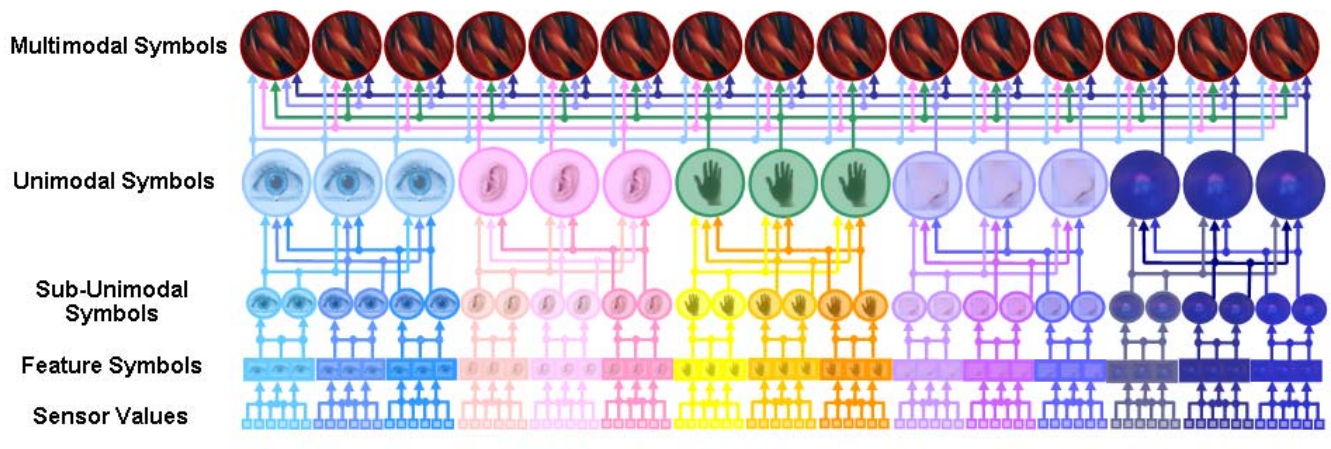

Visual Information Acoustic Information Tactile Information Olfactory Information Other Information

Fig. 4. Structure of a Neuro-symbolic Network

proposed model, the lowest-level connections are predefined. This means that it is fixed at initial system start up what feature symbols shall be extracted out of sensor data and how these feature symbols form subunimodal symbols. In contrast to the lower layers, correlations between higher layers can be learned from examples. Therefore, at initial system start-up, there do not exist connections between the sub-unimodal layer and the unimodal level and no connections between the unimodal level and the multimodal level. Suitable connections between these layers are learned by presenting a number of examples to the system, which cover all objects, events, and situations that the system shall perceive. Based on examples, there are set connections between sub-unimodal symbols and unimodal symbols in a first stage. After these connections have been set, there are calculated the correlation between the unimodal symbols and the multimodal symbols in a further step. Objects, events, and situations are considered as perceptual images and each of them is assigned to one particular neuro-symbol. For each object, event, and situation, a number of examples is necessary, because there can occur deviations in how they are represented by sensory data. Figure 5 illustrates the learning process between the sub-unimodal and the unimodal level of the tactile perceptual system. Similar to supervised learning in neural networks, there are presented input data and target data to the system.

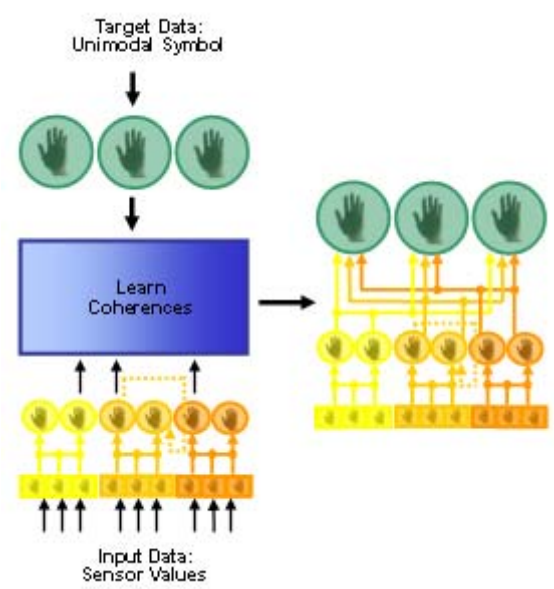

Fig. 5. Function Principle for Learning by Examples in Neuro-symbolic Networks
Input data are sensor data from sensors that are triggered when a certain situation occurs.

As the lower neuro-symbolic levels are already preconnected, based on these sensor data, certain lower level symbols are activated. The target data are the particular higher-level symbol that is assigned to the current situation occurring. During the learning phase, the system memorizes the examples. Based on these examples it extracts correlations that exist between lower-level and higher level symbols and sets connections between them accordingly. Besides forward connections from lower levels to higher levels, there can also exist feedback connections from higher levels downwards. These feedback connections can be determined and set by presenting the same examples as used for setting the forward connections to the system a second time. For an explanation of the necessity of feedback connections in perception see [30].

\section{B. Interaction between Perception and Other Cognitive Processes}

The neuro-symbolic information processing structure described until now can be regarded as the core of perception. The description given covers bottom-up information processing starting from sensor values. However, according to the last three points mentioned in section III when identifying characteristics of human perception, perception is also influenced from processes that are at least partly performed in areas lying outside the perceptual system of the brain. Perception is influenced and modified by memory, knowledge, and by focus of attention. Additionally, emotional evaluations are important to classify perceptual images and give subjective meaning to them (see figure 6).

Influence from Memory and Knowledge

According to [12], perception is influenced by knowledge and memory, in a top-down manner. Memory and knowledge cover factual knowledge, knowledge about the context where a situation occurs, past-experience of what happened before, and expectation. By integrating knowledge into the perception process, perception can be facilitated and ambiguous sensor data can be resolved. Unfortunately, neuroscientists and neuro-psychologists do not yet agree about the question how and on what level interaction between knowledge, memory, and perception takes place. 


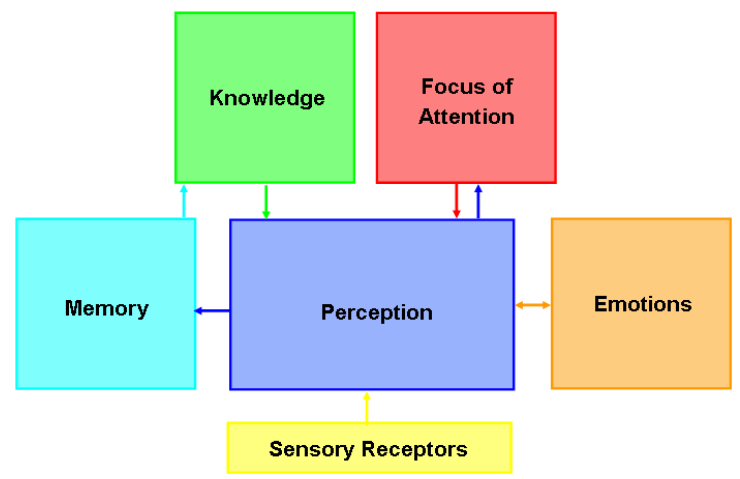

Fig. 6. Interaction between Perception and other Cognitive Processes

In the model, knowledge can influence the activation state of neuro-symbols. The interaction can principally take place on the sub-unimodal, unimodal, or the multimodal level. This means that the activation state of neurosymbols in these layers can be increased or decreased based on this cognitive information. Imagine for example that a room is monitored and the room is empty at the beginning. Now, the system can "know" that certain situations cannot occur as long as there does not enter a person. Therefore, neuro-symbols, which are correlated with activities performed by persons, cannot be activated. The event that a person enters a room only takes a very short time. Accordingly, the corresponding neuro-symbol is also activated only for a brief moment. Therefore, there needs to exist a mechanism to memorize that a person entered the room, because this activity has influence on future perception. In the proposed model, this task is performed by memory symbols. Memory symbols interact with knowledge and can principally receive information from the sub-unimodal, unimodal, or the multimodal level. They are set when a certain event happens in the environment and are reset when another event happens. Coming back to the example of an entering person, a memory symbol "person is present in the room" would be set if a person enters the room and would be reset if the person has left the room again.

\section{Influence from Focus of Attention}

If in an environment a number of situations happen in parallel, it can occur that lower-level symbols cannot be correctly assigned to higher level symbols. This effect can also be observed in human perception, if perception is overloaded. An overloading of perception means that there are too many perceptual stimuli present at a moment to integrate them all at once into a unified perception. In such a case, illusionary conjunctions occur. In the brain, a mechanism called focus of attention helps to correctly merge information coming from various sources. Similar as in human perception, such a mechanism of focus of attention also exists in the introduced model for humanlike perception. This mechanism constraints the spatial area in which perceptual images of different modalities are merged. In the model, focus of attention interacts with perception on the feature symbol level, which is the symbol level where neuro-symbols are topographic and therefore location dependent.

\section{Emotional Evaluation of Perceptual Images}

According to [27], besides the externally directed perception of the environment, there also exists an internal perception of the body by emotions. Emotions are akin to a sensory modality - an internally directed sensory modality that provides information about the current state of the body. Emotions are of importance for external perception, because they can be triggered from perceptual images of the outside world and can classify them as good or bad. Via a triggering of emotions, certain perceptual images can directly result in action tendencies. Therefore, a realization of emotions in technical systems can get important if human behavior shall be emulated. According to [5], it can be distinguished between primary emotions and secondary emotions. Primary emotions appear to consist in "hardwired" connections between certain external situations of biological significance and the subjective responses they evoke. Secondary emotions are "learned" connections between perceptual images and a response. In the presented model, it is possible to evaluate perceptual images represented as neuro-symbols by emotions represented as emotion symbols. In later development stages, these emotion symbols will provide an interface between the introduced perceptual system and an action system. In the model, there principally exist primary emotion symbols and secondary emotion symbols. Primary emotion symbols are already assigned to certain perceptual images at system start-up to support decision units using this model as a basis. Correlations between perceptual images and secondary emotion symbols have been designated to be learned from examples.

\section{Simulation And Testing}

To test and evaluate the model presented, the modeling language AnyLogic was used together with Java. The reason for choosing AnyLogic lies in the fact that this simulation environment offers certain design elements (active objects, ports, connectors, messages, interface and state variables, timers, state charts) that allow it to model and implement neuro-symbols. Besides this, a modular hierarchical design is possible and by using its graphic development environment, model development gets more comprehensible. These facts allow efficient testing. To test and evaluate the model, sensor data from various sources are necessary. For the provision of these sensor data, a simulator was used, which allows it to generate sensor values based on a virtual environment. [14], [15] This simulator is developed to simulate sensor values in order to perceive scenarios in a virtual office environment. The reason for simulating the sensor values is on the one hand the cost reduction for testing in comparison to real physical installations. On the other hand, the simulator allows it to evaluate which sensors are necessary to detect scenarios most effectively and efficiently and where they should be mounted. Simulation results show that processing of information in a modular hierarchical fashion considering the described bottom-up and topdown processes as well as feedbacks is an adequate way for handling end interpreting sensory data. 


\section{CONCLUSION AND OUTLOOK}

This paper suggested a model for emulating the perceptual system of the human brain to build next generation intelligent sensor fusion systems, e.g. for surveillance systems in buildings and to allow robots to perceive their environment autonomously. Therefore, a new information processing principle was introduced, which is called neuro-symbolic information processing. According to this method, sensory data are processed by so-called neurosymbolic networks to result in "awareness" of what is going on in an environment. The basic processing units of neuro-symbolic networks are neuro-symbols. Besides bottom-up information processing of sensory data, focus of attention, memory, and knowledge can influence the perceptive process. Additionally, there was suggested a mechanism to evaluate perceptual images by emotions, which provide an interface between perception and action. Emotions can be regarded as internally directed perception. Perceptual images coming from the external world represented by neuro-symbols are grounded in sensory receptors. To provide these sensor data, a simulator was used. To ground emotion symbols, they would have to be connected to a visceral body. This body could principally also be simulated. To build such a simulator, a quite detailed model about visceral processes in the body would be necessary. However, such a model is not yet available.

Nevertheless, besides the problem of symbol grounding for emotions, the proposed model provides a powerful and flexible tool for information processing of sensor data. By making certain adaptations, neuro-symbolic information processing could also be used for other application domains. What will be subject to further work is to develop a toolbox for neuro-symbolic networks comparable to the existing toolboxes for neural networks, which will allow fast and comfortable development and testing. With this toolbox, the method of neuro-symbolic information processing shall get attractive for a broader group of users.

\section{REFERENCES}

[1] X. Bian, G. D. Abowd, J. M. Rehg, "Using Sound Source Localization to Monitor and Infer Activities in the Home," GVU Technical Reports, 2004.

[2] J. Beyerer, F. P. León, K. Sommer, "Informationfusion in der Messund Sensortechnik“, Universitätsverlag Karlsruhe, 2006.

[3] W. Burgstaller, "Interpretation of Situations in Buildings," Ph.D. dissertation, Vienna University of Technology, 2007.

[4] E. Datteri, G. Teti, C. Laschi, G. Tamburrini, P. Dario, C. Guglielmelli, "Expected Perception: An Anticipation-Based Perception-Action Scheme in Robots," in Proc. International Conference on Intelligent Robots and Systems, 2003, pp. 934-939.

[5] A. Damasio, "Descartes' Error - Emotion, Reason, and the Human Brain," Penguin Books, 1994.

[6] J. Davis, "Biological Sensor Fusion Inspires Novel System Design," in Proc. Joint Service Combat Identification Systems Conference, 1997.

[7] D. P. W. Ellis, "Prediction-driven Computational Auditory Scene Analysis," Ph.D dissertation, Massachusetts Institute of Technology, 1996.

[8] W. Elmenreich, "Sensor Fusion in Time-Triggered Systems," Ph.D dissertation, Vienna University of Technology, 2002.
[9] W. Elmenreich, “A Review on System Architectures for Sensor Fusion Applications," International Federation for Information Processing, 2007, pp. 547-559.

[10] M. O. Ernst, H. H. Bülthoff, "Merging the Senses into a Robust Percept,".TRENDS in Cognitive Sciences, 2004, Vol. 8, pp. 162 169.

[11] R. M. French, "Review of The Engine of Reason, the Seat of the Soul," in Minds \& Machines, 1996, Vol. 6, pp. 416-421.

[12] E. B. Goldstein, "Wahrnehmungspsychologie," Spektrum Akademischer Verlag, 2002.

[13] S. O. Götzinger, "Scenario Recognition based on a Bionic Model for Multi-Level Symbolization," Master Thesis, Vienna University of Technology, 2006.

[14] H. Hareter, G. Pratl, D.Bruckner, "A Simulation and Visualization System for Sensor and Actuator Data Generation," in Proc. IFAC, 2005, pp.

[15] H. Hareter, "Worst Case Szenarien Simulator für die Gebäudeautomation,",Ph.D. dissertation, Vienna University of Technology, 2008

[16] R. Harvey, K. Heinemann, "Biological Vision Models for Sensor Fusion," in Proc. 1st IEEE Conference on Control Applications, 1992, pp. 392-397.

[17] B. Hommel, B. Milliken, "Taking the Brain Serious: Introduction to, in, and across Perception and Action," Psychological Research, 2005.

[18] D. Joyce, L. Richards, A. Cangelosi, K. R. Coventry, "On the Foundations of Perceptual Symbol Systems: Specifying Embodied Representations via Connectionism," in Proc. 5th International Conference on Cognitive Modeling, 2003, pp. 147-152.

[19] R. C. Luo, M. G. Kay, "Multisensor Integration and Fusion in Intelligent Systems," IEEE Transactions on Systems, Man, and Cybernetics, 1989, Vol. 19, pp. 901-931.

[20] A. R. Luria, "The Working Brain - An Introduction in Neuropsychology,” Basic Books, 1973.

[21] L. I. Perlovsky, B. Weijers, C. W. Mutz "Cognitive Foundations for Model-based Sensor Fusion," in Proc. of SPIE, 2003, Vol. 5096, pp. 494-501.

[22] G. Pratl, "Processing and Symbolization of Ambient Sensor Data" Ph.D. dissertation, Vienna University of Technology, 2006.

[23] A. Richtsfeld, "Szenarienerkennung durch symbolische Datenverarbeitung mit Fuzzy-Logic," Master Thesis, Vienna University of Technology, 2007.

[24] A. L. Roskies, "The Binding Problem," Neuron, Vol. 24, 1999, pp. 7-9.

[25] H. Ruser, F. P. León, "Informationfusion - Eine Übersicht," Technisches Messen Oldenbourg Verlag, 2007, Vol. 74, pp. 93-102

[26] J. Sillanpää, A. Klapuri, J. Seppäne, T. Virtanen, "Recognition of Acoustic Noise Mixtures by Combined Bottom-up and Top-down Processing," in Proc. European Signal Processing Conference EUSIPCO, 2000, Vol. 1, pp. 335-338.

[27] M. Solms O. Turnbull, "The Brain and the Inner World - An Introduction to the Neuroscience of Subjective Experience," Other Press New York, 2002

[28] J. van Dam, "Environment Modelling for Mobile Robots: Neural Learning for Sensor Fusion," Ph.D. dissertation, University of Amsterdam, 1998.

[29] R. Velik, “A Multi-sensory, Symbolic, Knowledge-based Model for Humanlike Perception," in Proc 7th International Conference on Fieldbus \& Networks in Industrial \& Embedded Systems, 2007.

[30] R. Velik, "A Model for Multimodal Humanlike Perception based on Modular Hierarchical Symbolic Information Processing, Knowledge Integration, and Learning," in Proc. Bionetics, 2007. 